\title{
Nesfatin-1 and caspase-cleaved cytokeratin-18: Promising biomarkers for Alzheimer's disease?
}

\author{
Alpua $\mathrm{M}^{1}, \mathrm{Kisa} \mathrm{U}^{2}$ \\ Department of Neurology, Kirikkale University, Faculty of Medicine, Kirikkale, Turkey. \\ dr.muratalpua@yahoo.com
}

\section{ABSTRACT}

OBJECTIVES: To investigate the use of nesfatin-1 and caspase-cleaved cytokeratin-18 serum levels as biomarkers in Alzheimer's disease.

METHODS: The study group consisted of 39 patients with Alzheimer's disease (AD) and 39 controls. Demographic characteristics including gender, age, body mass index, mini-mental status examination (MMSE) and duration of disease were recorded. The ELISA method was used to measure serum nesfatin-1 and CCCK-18 levels in serum samples. RESULTS: Serum nesfatin-1 levels were statistically significantly higher in the AD patient group than in controls. There was no significant difference between the groups with regards to serum CCCK-18 levels. Pearson analysis showed no significant correlation between serum nesfatin-1, serum CCCK-18 levels, mini-mental status examination and disease duration.

CONCLUSION: This study proved that serum nesfatin-1 levels can be used as a biomarker in Alzheimer's disease by showing a statistically significant high level of serum nesfatin-1 in patients with Alzheimer's disease. This is the first study to suggest that nesfatin-1 can be used as a biomarker in Alzheimer's disease. In addition, our study showed that CCCK-18 can be used as a prognostic biomarker for Alzheimer's disease. Further comprehensive studies should be done to clarify the use of serum nesfatin-1 and CCCK-18 levels as biomarkers for Alzheimer disease (Tab. 3, Fig. 2, Ref. 25). Text in PDF www.elis.sk.

KEY WORDS: nesfatin-1, CCCK-18, Alzheimer's disease, mini-mental status examination.

\section{Introduction}

Due to the increase in the elderly population worldwide, one of the significant public health problems around the world is dementia. According to data from the world health organization, the number of dementia patients is expected to triple by 2050 . Alzheimer's disease (AD), which represents more than $65 \%$ of all dementia cases, is the most common primary cause of dementia (1). AD can only be definitively diagnosed by autopsy because the expression of brain plaques and neurofibrillary tangles cannot be fully captured by existing imaging technologies. However, Alzheimer's dementia can be clinically diagnosed using clinical guidelines and exclusion of other diseases that may lead to dementia. Tau phosphorylation and amyloid $\beta(\mathrm{A} \beta)$ accumulation are characteristic pathophysiologic signs involved in the pathogenesis of $\mathrm{AD}$; several studies have been conducted to develop diagnostic tests based on these pathologies. Mulder et al have demonstrated that AD can be detected with high sensitivity by

${ }^{1}$ Department of Neurology, Kirikkale University, Faculty of Medicine, Kirikkale, Turkey, and ${ }^{2}$ Department of Biochemistry, Kirikkale University, Faculty of Medicine, Kirikkale, Turkey

Address for correspondence: M. Alpua, Kirikkale University, Faculty of Medicine, Department of Neurology, Kirikkale, Postal code: 71450, Turkey. Phone: $+90.318 .3335000 / 5056$, Fax: +90.318 .2240786$

Acknowledgement: This study was funded by Kirikkale University with this title Financial support and sponsorship. measuring concentrations of protein biomarkers such as $A \beta 1-42$, total-tau, and phospho-tau in the cerebral spinal fluid (CSF) (2). These markers showed high diagnostic potential, and are widely used for experimental and diagnostic purposes. On the other hand, this examination is invasive and not suitable for everyday use in outpatient clinics. The use of peripheral markers such as $A B$ and tau as a diagnostic tool in easily accessible peripheral cells, such as in platelets and skin fibroblasts, has been investigated for many years (3). As a result, a new biomarker that is non-invasive, faster and more economical is needed.

Nesfatin-1, an 82-amino acid peptide, is excreted in various brain regions including the arcuate nucleus, supraoptic nucleus, hypothalamic paraventricular nucleus, lateral hypothalamic area, and nucleus tractus solitarii (4) and is defined primarily for regulating food intake (5). It can pass through the blood-brain barrier in both directions. It has already been proposed as a potential diagnostic tool for many diseases due to its stability and suitability in the serum. But there is no relevant study of the use of nesfatin-1 as a biomarker in Alzheimer's disease. Because AD is a disease caused by global brain atrophy and nesfatin is also produced in the brain, we suggested that nesfatin could be used as a biomarker in AD.

Apoptosis is a form of cell death that must be followed regularly and occurs when an organism is functioning normally. Apoptosis is also an important factor in neurodegenerative diseases. It plays a significant role in neuronal cell death in $\mathrm{AD}(6)$. During apoptosis, cytokeratin-18, which is derived from epithelial and parenchymal cells, is disrupted by the action of caspases. Following this frag- 
mentation, caspase-cleaved cytokeratin-18 (CCCK-18) is released into the peripheral blood (7). Therefore, CCCK-18 is an apoptotic biomarker (8). It has been suggested that higher serum concentrations of CCCK-18 are associated with poor prognosis or mortality in severe sepsis, breast cancer and chronic hepatitis (9-11). But there is no study investigating the use of CCCK-18 as a biomarker in AD. Because CCCK-18 is an apoptotic biomarker and apoptosis plays a significant role in the pathogenesis of $\mathrm{AD}$, we suggested that CCCK-18 could be used as a prognostic biomarker for AD.

\section{Material and methods}

We chose our patients from among the patients of the Kirikkale University Medical Faculty Neurology Clinic. The doctrines of the actual version of the Helsinki Declaration were followed; approval from the institutions' ethical committee was obtained, and the protocol of the study was explained to the patients and patients' relatives. After receiving detailed information, each patient or relative signed an informed consent form.

Thirty-nine adults with the clinical diagnosis of AD and 39 healthy controls were involved in the study. All AD patients were diagnosed based on the National Institute on Aging (NIA) and Alzheimer's Association criteria. The patients were followed-up in our outpatient clinic at regular intervals for at least 1 year. The mean disease duration of AD patients was 3 years (range: $1-7$ years). All $\mathrm{AD}$ patients were receiving cholinesterase inhibitors and/or memantine. All patients were in a stable phase of the disease; there was no delirium or infection. Our patient population was selected as a sample for our investigation. To the extent possible, sex- and age-matched healthy volunteer workers without a history of neurodegenerative and/or psychiatric diseases, were taken as controls. Previous similar studies were also taken into consideration.

Demographic characteristics, including gender, age, body mass index and mini-mental exam scale, and disease course were recorded.

Exclusion criteria were as follows: history of any vascular or systemic disease, any other neurodegenerative disease, any psychiatric disease, epilepsy, and substance or drug abuse.

Blood samples were collected intravenously from both healthy controls and patients. There were no cases of infection or delirium in the patients when the samples were taken. Serum was separated from the blood samples by centrifugation at $1,000 \mathrm{~g}$ for $20 \mathrm{~min}$ and kept frozen at $-80^{\circ} \mathrm{C}$ until used for analyses to measure nesfatin-1 and CCCK-18 levels. Serum nesfatin-1 levels were measured by Human Nesfatin-1 ELISA Kit (Cuasabio Technology, Houston, USA) with the enzyme-labeled immunometric assay method (reference interval: $31.25-2000 \mathrm{pg} / \mathrm{ml}$ ). Serum CCCK-18 levels were measured by M30 Apoptosense ELISA Kit (Diapharma Group, West Chester, OH, USA) with the enzyme-labeled immunometric assay method (reference interval: 0-1000 U/L).

After taking the blood samples, each patient's regular followup continued for at least 1 year at our outpatient clinic.

SPSS version 16.0 was used to analyze the results. A p-value $<$ 0.05 was considered to indicate statistical significance. Categorical variables were expressed as proportions. Continuous variables
Tab. 1. The demographics and Nesfatin-1 and CCCK-18 levels of patients with AD and control group.

\begin{tabular}{lccc}
\hline & $\begin{array}{c}\text { Patients } \\
\text { mean } \pm \text { SD } \\
(\mathrm{n}=39)\end{array}$ & $\begin{array}{c}\text { Controls } \\
\text { mean } \pm \text { SD } \\
(\mathrm{n}=39)\end{array}$ & $\mathrm{p}$ \\
\hline Age, $\mathrm{y}$ & $78.5 \pm 6.5$ & $68.2 \pm 8.8$ & 0.17 \\
\hline Gender $($ female/male $)$ & $\begin{array}{c}18 / 21 \\
(46.2 \% / 53.8 \%)\end{array}$ & $\begin{array}{c}18 / 21 \\
(46.2 \% / 53.8 \%)\end{array}$ & 1.000 \\
\hline BMI $\left(\mathrm{kg} / \mathrm{m}^{2}\right)$ & $24.48 \pm 1.73$ & $24.20 \pm 1.32$ & 0.176 \\
\hline AD duration, $\mathrm{y}$ & $3.3 \pm 1.5$ & & \\
\hline MMSE $(\mathrm{median})$ & $18(7-23)$ & & \\
\hline Nesfatin-1 $(\mathrm{pg} / \mathrm{ml})$ & $200.4 \pm 191.7$ & $113.5 \pm 145.8$ & 0.001 \\
\hline CCCK-18 $(\mathrm{U} / \mathrm{L})$ & $225.8 \pm 338.0$ & $159.8 \pm 61.4$ & 0.269 \\
\hline
\end{tabular}

$\mathrm{AD}$ - Alzheimer disease, MMSE - mini-mental scale examination, BMI - body mass index

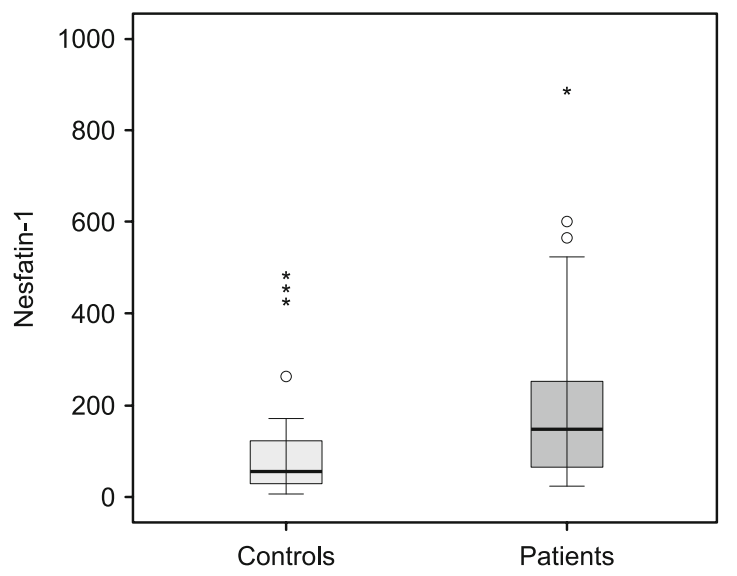

Fig. 1. The mean value and standard deviation of Nesfatin-1 levels in patients and controls.

were presented as mean $\pm \mathrm{SD}$. The $\chi^{2}$ test was used to test differences in categorical values and Student's t-test for continuous variables. Association of nesfatin-1 and CCCK-18 with minimental status examination and disease duration were examined using Spearman's correlation in all subjects.

\section{Results}

Table 1 summarizes the characteristics of the subjects. The mean sex, and age distribution and body mass index in $\mathrm{AD}$ patients did not differ from those of controls. Serum nesfatin-1 levels were significantly higher in $\mathrm{AD}$ patients than in the control group $(\mathrm{p}=$ 0.001) (Fig. 1). Spearman's analysis did not show any significant correlation between mini-mental status scale, disease duration and nesfatin-1 levels (Tab. 2). Figure 2 shows the sensitivity and specificity of nesfatin-1 values in ROC analysis. The confidence intervals around nesfatin-1 values are mentioned in Table 3.

\section{Discussion}

AD causes global brain atrophy and severe cognitive impairment. As such, it is an important cause of morbidity and mortality at an advanced age. The diagnosis of AD is clinically established 
Tab. 2. The correlations between MMSE, disease duration and Nesfatin-1 levels.

\begin{tabular}{lcc}
\hline Spearman's rho & \multicolumn{2}{c}{ MMSE } \\
\cline { 2 - 3 } & Correlation coefficient & $\mathrm{p}$ \\
\hline Nesfatin 1-MMSE & -0.092 & 0.578 \\
Disease duration-MMSE & -0.608 & $<0.001$ \\
Nesfatin 1-disease duration & 0.191 & 0.244 \\
\hline
\end{tabular}

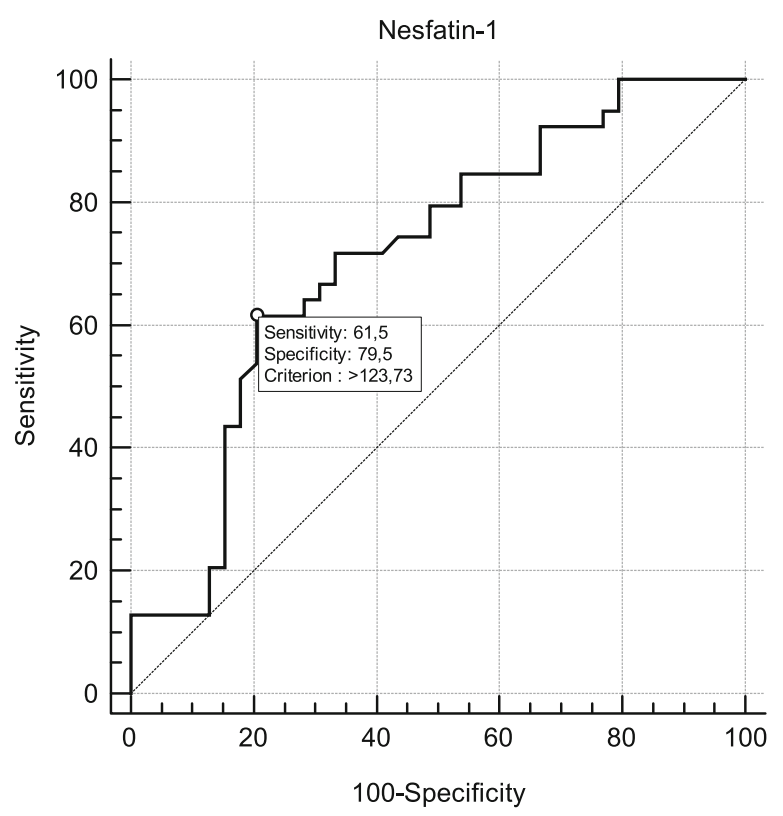

Fig. 2. The ROC analysis of Nesfatin 1 values.

according to current criteria. Many different neuropsychological tests are used in the clinical diagnosis of the disease. Besides that, many diagnostic biomarkers have been investigated for $\mathrm{AD}$, and some have been used in clinical practice (12). However, these are usually CSF biomarkers and the market values of these biomarkers are also very high. The facts that nesfatin- 1 is easily detectable in peripheral blood and the cost of the nesfatin-1 kits are relatively more convenient for daily practice are the reasons why nesafatin-1 is an important biomarker candidate for $\mathrm{AD}$.

In our present study, we demonstrated serum nesfatin-1 levels to be significantly higher in $\mathrm{AD}$ patients compared to healthy controls. Previous studies have suggested that nesfatin-1 may exhibit an anti-inflammatory effect in the central nervous system (CNS). For example, Ozsavci et al reported that nesfatin-1 treatment suppressed subarachnoid hemorrhage-induced elevations in the plasma levels of proinflammatory cytokines in rats (13). The authors also suggested that nesfatin-1 has an antiapoptotic effect in CNS. Besides that, Tang et al also demonstrated the anti-apoptotic and anti-inflammatory effects of nesfatin-1 in CNS. Their study demonstrated that the administration of nesfatin- 1 diminished caspase- 3 activity and reduced apoptotic nervous cells in post-traumatic rats (14). It is important to note that inflammation and apoptosis play a significant role in the pathophysiology of AD. Therefore, the detection of high serum nesfatin-1 levels in Alzheimer's disease is important and this finding may be an indirect indicator of the
Tab. 3. The confidence intervals around nesfatin-1 values.

\begin{tabular}{|c|c|c|c|c|}
\hline \multicolumn{5}{|c|}{ Group } \\
\hline \multirow{14}{*}{$\begin{array}{l}\text { I } \\
\text { 売 } \\
\text { 莺 } \\
\text { Z }\end{array}$} & \multirow{7}{*}{ 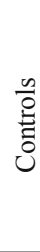 } & mean & & 113.4679 \\
\hline & & \multirow{2}{*}{$\begin{array}{l}95 \% \text { confidence interval for } \\
\text { mean }\end{array}$} & lower bound & 66.2028 \\
\hline & & & upper bound & 160.7331 \\
\hline & & median & & 54.4600 \\
\hline & & std. deviation & & 145.80700 \\
\hline & & minimum & & 5.72 \\
\hline & & maximum & & 489.88 \\
\hline & \multirow{7}{*}{ 营 } & mean & & 200.3528 \\
\hline & & $95 \%$ confidence interval for & lower bound & 138.2175 \\
\hline & & mean & upper bound & 262.4881 \\
\hline & & median & & 145.9700 \\
\hline & & std. deviation & & 191.67952 \\
\hline & & minimum & & 22.39 \\
\hline & & maximum & & 893.84 \\
\hline
\end{tabular}

anti-apoptotic and anti-inflammatory effects of this peptide. At the same time, this finding also suggests that nesfatin- 1 can be used as a biomarker for AD.

$\mathrm{AD}$ and Parkinson's disease are the most common neurodegenerative diseases in the elderly. Although the underlying pathologies are different, the common feature of both diseases lies in the loss of neuronal cells. Zhen et.al suggested that nesfatin-1 has a neuroprotective effect by preventing neuronal cell loss, caspase 3 activation and mitochondrial dysfunction in dopaminergic cells (15). A similar neuroprotective effect may be present in AD, and due to this potential effect, serum levels of nesfatin-1 were found to be higher in Alzheimer's patients in our study.

It is known that spatial memory impairments may occur already in the early stages of $\mathrm{AD}(16)$. These impairments are associated with the reduction of excitatory glutamatergic terminals (17). Synaptic loss in the brain especially in the hippocampus reduces the ability to acquire spatial information (18). In a recent study, Erfani et al showed that nesfatin-1 improves spatial memory impairment by inhibiting microglial and caspase- 3 activation. In another study, it has been shown that nesfatin-1 significantly reduces apoptosis and necrotic cell death in the hippocampus (19). Therefore these valuable findings may explain why serum nesfatin-1 levels are higher in Alzheimer's patients than in controls.

The deterioration in learning and memory functions arises from the early stages of $\mathrm{AD}$. The anatomical structures that are most clearly associated with learning and memory are the hippocampus and prefrontal cortex. From the early stages of AD, it is known that pathological findings occur in these anatomical structures. It is also known that nesfatin- 1 is widely expressed in these anatomical structures in the brain (20). In a previous study, Chen et al suggested that nesfatin- 1 has a moderating effect on learning and memory in rats (21) and from this point of view, higher serum levels of nesfatin-1 in Alzheimer's patients may be associated with this effect.

In this study, we showed that serum CCCK-18 levels are similar in Alzheimer disease patients compared to healthy controls. Our study is the first one to evaluate whether CCCK-18 can be used as a biomarker in AD. In previous studies, high levels of CCCK-18 have been associated with early mortality as well as 
late high mortality in various conditions. For example, Lorente et al showed that there is an association between early mortality and higher serum CCCK-18 levels in severe spontaneous intracerebral hemorrhage patients (22). They also showed that there are higher serum CCCK-18 levels in cases of severe malignant middle cerebral artery infarction, severe sepsis and severe traumatic brain injury $(6,23,24)$. Besides that, Gu et al suggested that there is an association between increased serum CCCK-18 concentrations and late mortality in intracerebral hemorrhage (25). The facts that there was no statistically significant increase in CCCK-18 values in our patients, and at the same time no mortality occurred among the patients followed up for a year indicate that CCCK-18 levels can be used as a prognostic biomarker in AD.

There was a limitation that could have affected the results of our study, namely the number of participants was relatively low. This was because our hospital serves a relatively small population. Further large-scale studies could give more precise information in the future.

\section{Conclusion}

Our current study suggested that nesfatin- 1 could be used as a biomarker for $\mathrm{AD}$ as well as that it could play a possible anti-inflammatory and neuroprotective role. Beside that, this study is the first study showing that nesfatin-1 can be used as a biomarker in AD. Furthermore, this study shows that serum CCCK-18 levels can be used as a prognostic indicator in $\mathrm{AD}$. We suggest that further comprehensive studies may clarify the details of the biomarking role of nesfatin-1 and prognostic role of CCCK-18 in AD in the future.

\section{References}

1. Rizzi L, Rosset I, Roriz-Cruz M. Global epidemiology of dementia: Alzheimer's and vascular types. Biomed Res Int 2014; 2014: 908915.

2. Mulder C, Verwey NA, van der Flier WM, Bouwman FH, Kok A, van Elk EJ et al. Amyloid-beta (1-42), total tau, and phosphorylated tau as cerebrospinal fluid biomarkers for the diagnosis of Alzheimer disease. Clin Chem 2010; 56: 248-253.

3. Malaplate-Armand C, Desbene C, Pillot T, Olivier JL. Biomarkers for early diagnosis of Alzheimer's disease: current update and future directions. Rev Neurol (Paris) 2009; 165: 511-520.

4. Chen X, Dong J, Jiang ZY. Nesfatin-1 influences the excitability of glucosensing neurons in the hypothalamic nuclei and inhibits the food intake. Regul Pept 2012; 177: 21-26.

5. Sahpolat M, Ari M. Plasma nesfatin 1 level in patients with first attack psychosis. Bratisl Lek Listy 2017; 118 (2): 77-79.

6. Eckert A, Marques CA, Keil U et al. Increased apoptotic cell death in sporadic and genetic Alzheimer's disease. Ann N Y Acad Sci 2003; 1010 : 604-609.

7. Lorente $\mathbf{L}$ et al. Serum levels of caspase-cleaved cytokeratin-18 and mortality are associated in severe septic patients: pilot study. PLoS One 2014; 9: e109618.

8. Soleiman A, Lukschal A, Hacker S et al. Myocardial lipofuscin-laden lysosomes contain the apoptosis marker caspase-cleaved cytokeratin-18. Eur J Clin Invest 2008; 38: 708-712.
9. Sumer S, Aktug Demir N, Kölgelier S, Cagkan Inkaya A, Arpaci A, Saltuk Demir L et al. (2013) The Clinical Significance of Serum Apoptotic Cytokeratin 18 Neoepitope M30 (CK-18 M30) and Matrix Metalloproteinase 2 (MMP-2) Levels in Chronic Hepatitis B Patients with Cirrhosis. Hepat Mon 13: e10106.

10. Ueno T, Toi M, Bivén K, Bando H, Ogawa T, Linder S. Measurement of an apoptotic product in the sera of breast cancer patients. Eur J Cancer 2003; 39: 769-774.

11. Moore DJ, Greystoke A, Butt F, Wurthner J, Growcott J, Hughes A et al. A pilot study assessing the prognostic value of CK18 and nDNA biomarkers in severe sepsis patients. Clin Drug Investig 2012; 32: 179-187.

12. Counts SE, Ikonomovic MD, Mercado N, Vega IE, Mufson EJ. Biomarkers for the Early Detection and Progression of Alzheimer's Disease. Neurotherapeutics 2017; 14: 35-53.

13. Ozsavci D, Ersahin M, Sener A et al. The novel function of nesfatin-1 as an anti-inflammatory and antiapoptotic peptide in subarachnoid hemorrhageinduced oxidative brain damage in rats. Neurosurgery 2011; 68: 1699-1708.

14. Tang CH, Fu XJ, Xu XL et al. The anti-inflammatory and antiapoptotic effects of nesfatin-1 in the traumatic rat brain. Peptides 2012; 36: 39-45.

15. Tan Z, Xu H, Shen X, Jiang H. Nesfatin-1 antagonized rotenone-induced neurotoxicity in MES23.5 dopaminergic cells. Peptides 2015; 69: 109-114.

16. Chapman PF et al. Impaired synaptic plasticity and learning in aged amyloid precursor protein transgenic mice. Nat Neurosci 1999; 2: 271-276.

17. Hsia AY, et al. Plaque-independent disruption of neural circuits in Alzheimer's disease mouse models. Proc. Natl Acad. Sci. USA. 1999; 96:32283233.

18. Scheff SW, Price DA, Schmitt FA, DeKosky ST, Mufson EJ. Synaptic alterations in CA1 in mild Alzheimer disease and mild cognitive impairment. Neurology 2007; 68: 1501-1508.

19. Erfani S, Moghimi A, Aboutaleb N, Khaksari M. Protective effects of Nesfatin-1 peptide on cerebral ischemia reperfusion injury via inhibition of neuronal cell death and enhancement of antioxidant defenses. Metab Brain Dis 2018; 30.

20. Goebel-Stengel M, Wang L, Stengel A, Tache Y. Localization of nesfatin-1 neurons in the mouse brain and functional implication. Brain Res 2011; 1396: 20-34.

21. Chen Z, Xu YY, Wu R, Han YX, Yu X, Ge JF, Chen FH. Impaired learning and memory in rats induced by a high-fat diet: involvement with the imbalance of nesfatin- 1 abundance and copine 6 expression. J Neuroendocrinol 2017; 29.

22. Lorente L, Martín MM, Pérez-Cejas A et al. Association between serum levels of caspase-cleaved cytokeratin-18 and early mortality in patients with severe spontaneous intracerebral hemorrhage. BMC Neurosci 2018; 19 (1): 23.

23. Lorente L, Martín MM, Pérez-Cejas A, Ramos L, Argueso M et al. High serum levels of caspase-cleaved cytokeratin-18 are associated with malignant middle cerebral artery infarction patient mortality. BMC Neurol 2018; 18 (1): 32 .

24. Lorente L, Martin MM, Gonzalez-Rivero AF, Argueso M, Ramos L et al. Serum levels of caspase-cleaved cytokeratin-18 in patients with severe traumatic brain are associated with mortality: a pilot study. PLoS One 2015; 10 (3): e0121739.

25. Gu SJ, Lu M, Xuan HF, Chen XZ, Dong WF, Yan XF et al. Predictive value of serum caspase-cleaved cytokeratin-18 concentrations after acute intracerebral hemorrhage. Clin Chim Acta 2016; 452: 124-128.

Received January 3, 2019. Accepted February 6, 2019. 